\title{
Prediction of Non-canonical Routes for SARS-CoV-2 Infection in Human Placenta Cells
}

\author{
Flávia Bessi Constantino ${ }^{1 \dagger}$, Sarah Santiloni Cury ${ }^{1 \dagger}$, Celia Regina Nogueira ${ }^{2}$, \\ Robson Francisco Carvalho ${ }^{1 *}$ and Luis Antonio Justulin ${ }^{1 *}$ \\ ${ }^{1}$ Department of Structural and Functional Biology, Institute of Biosciences, São Paulo State University (UNESP), Botucatu, Brazil, \\ ${ }^{2}$ Department of Internal Clinic, Botucatu Medicine School, São Paulo State University (UNESP), Botucatu, Brazil
}

OPEN ACCESS

Edited by: James Leland Olds, George Mason University, United States

Reviewed by: Estela Bevilacqua, University of São Paulo, Brazil Arvind Ramanathan, Argonne National Laboratory (DOE), United States

*Correspondence:

Luis Antonio Justulin l.justulin@unesp.br

Robson Francisco Carvalho robson.carvalho@unesp.br

${ }^{+}$These authors have contributed equally to this work

Specialty section:

This article was submitted to Biological Modeling and Simulation, a section of the journal Frontiers in Molecular Biosciences

Received: 06 October 2020

Accepted: 15 October 2021 Published: 08 November 2021

Citation:

Constantino FB, Cury SS, Nogueira $C R$, Carvalho RF and Justulin LA (2021) Prediction of Noncanonical Routes for SARS-CoV-2 Infection in Human Placenta Cells.

Front. Mol. Biosci. 8:614728. doi: 10.3389/fmolb.2021.614728
The SARS-CoV-2 is the causative agent of the COVID-19 pandemic. The data available about COVID-19 during pregnancy have demonstrated placental infection; however, the mechanisms associated with intrauterine transmission of SARS-CoV-2 is still debated. Intriguingly, while canonical SARS-CoV-2 cell entry mediators are expressed at low levels in placental cells, the receptors for viruses that cause congenital infections such as the cytomegalovirus and Zika virus are highly expressed in these cells. Here we analyzed the transcriptional profile (microarray and single-cell RNA-Seq) of proteins potentially interacting with coronaviruses to identify non- canonical mediators of SARS-CoV-2 infection and replication in the placenta. Despite low levels of the canonical cell entry mediators ACE2 and TMPRSS2, we show that cells of the syncytiotrophoblast, villous cytotrophoblast, and extravillous trophoblast co-express high levels of the potential noncanonical cell-entry mediators DPP4 and CTSL. We also found changes in the expression of DAAM1 and PAICS genes during pregnancy, which are translated into proteins also predicted to interact with coronaviruses proteins. These results provide new insight into the interaction between SARS-CoV-2 and host proteins that may act as non-canonical routes for SARS-CoV-2 infection and replication in the placenta cells.

Keywords: COVID-19, SARS-CoV-2, placenta, virus entry mediator, DPP4, CTSL, DAAM1, PAICS

\section{INTRODUCTION}

The severe acute respiratory syndrome coronavirus 2 (SARS-CoV-2) is the causative agent of the coronavirus disease 2019 (COVID-19) (Wu et al., 2020). It was first notified at the end of 2019, in Wuhan, China, and became a worldwide pandemic (Dong E. et al., 2020). At the beginning of September 2021, COVID-19 infected over 227 million people and is the cause of approximately 4.674.673 deaths worldwide (https://coronavirus.jhu.edu/).

Older age, laboratory abnormalities, and several comorbidities are associated with the more severe cases of COVID-19 (Williamson et al., 2020). For specific groups of COVID-19 patients, for example, pregnant women, the potential impacts of SARS-CoV-2 infection remain mostly unknown, and data are limited. However, considering previous works reporting coronaviruses infections (Schwartz and Graham, 2020), pregnant women are at higher risk of SARS-CoV-2 infection due to physiological changes in the immune, cardiorespiratory, and metabolic systems (Qadri and Mariona, 2020).

Although only a small number of maternal virus infections are transmitted to the fetus, some may cause life-threatening diseases (Pereira, 2018). These viruses use cellular host entry mediators expressed by placenta cells, as described for the cytomegalovirus and the Zika virus (Pierson and 
Diamond, 2018; Hashimoto et al., 2020), to infect these cells. The Zika virus (ZIKV) outbreak, associated with fetal brain damage, emphasizes the necessity of further characterization and understanding of placental infection or intrauterine (vertical) transmission of SARS-CoV-2, as well as the possible adverse fetal outcomes. The few studies on the subject have provided contradictory findings, with some reports suggesting no evidence of placental infection or vertical transmission of SARS-CoV-2 (Celik et al., 2020; Chen et al., 2020; Yang and Liu, 2020). Conversely, multiple lines of evidence have shown placental SARS-CoV-2 infection in pregnant women diagnosed with moderate to severe COVID-19 (Schwartz and Morotti, 2020; Shende et al., 2021; Valdespino-Vázquez et al., 2021). Moreover, neonates born from mothers with COVID-19 presented a positive serological test for SARS-CoV-2 immunoglobulin (Ig) M and IgG (Dong L. et al., 2020; Zeng et al., 2020). While the IgG can be transferred from mother to fetus across the placenta, the detection of IgM in newborns suggests a vertical transmission of the virus, since IgM cannot cross the placental barrier due to its high molecular mass (Kimberlin and Stagno, 2020). Accordingly, SARS-CoV-2 RNA transmission was comprehensively confirmed by pathological and virological investigations (Patanè et al., 2020). Also, it was recently shown SARS-CoV-2 particles in syncytiotrophoblast with generalized inflammation, diffuse perivillous fibrin depositions, and tissue damage in an asymptomatic woman (Schoenmakers et al., 2020). Remarkably, these placental alterations due to the SARS-CoV2 infection lead to fetal distress and neonatal multi-organ failure. These results highlight the importance of exploring the expression profile of potential host mediators of the SARSCoV-2 that may create a permissive microenvironment to placental infection and enable vertical transmission of the virus.

In fact, like other viruses, SARS-CoV-2 requires diverse host cellular factors for infection and replication. The angiotensinconverting enzyme 2 (ACE2) is the canonical receptor for the SARS-CoV-2 spike protein receptor-binding domain (RBD) for viral attachment (Letko et al., 2020). This process is followed by $S$ protein priming by cellular transmembrane serine protease 2 (TMPRSS2) that allows the fusion of the virus with host cellular membranes (Letko et al., 2020). Single-cell RNA sequencing (scRNA-Seq) has demonstrated that both ACE2 and TMPRSS2 are co-expressed in multiple tissues affected by COVID-19, including airway epithelial cells, cornea, digestive and urogenital systems (Sungnak et al., 2020). Few cells express ACE2 and TMPRSS2 in the placenta (Pique-Regi et al., 2020; Sungnak et al., 2020), suggesting that SARS-CoV-2 is unlikely to infect the placenta through the canonical cell entry mediators. Therefore, other host interacting proteins may play a role in the biological cycle of the virus and contribute to the pathogenesis of SARS-CoV-2 in the placenta. In this paper, we demonstrate, through transcriptomic (microarray and scRNA-Seq) analysis and in silico predictions of virus-host protein-protein interactions, that cells of the syncytiotrophoblast, villous cytotrophoblast, and extravillous trophoblast express high levels of potential non-canonical cell-entry mediators dipeptidyl peptidase $4(D P P 4)$ and cathepsin L (CTSL), despite low-levels of ACE2 and TMPRSS2. We also found changes in the expression of DAAM1 and PAICS genes (translated into proteins predicted to interact with coronaviruses proteins) during pregnancy, which co-express with DPP4 and CTSL in placenta single cells (syncytiotrophoblast, villous cytotrophoblast, and extravillous trophoblast). These results open new avenues of investigation of the human placenta infection by the SARSCoV-2.

\section{RESULTS}

\section{Prediction of Non-canonical Routes for SARS-CoV-2 Infection in Human Placenta Cells}

We first investigated the gene expression in placental tissues of classical host-virus interacting proteins described in the literature. The canonical entry receptors ACE2 and TMPRSS2 were low expressed throughout gestation. CTSL, which is translated into a lysosomal cysteine proteinase that plays a role in intracellular protein catabolism - presented the highest level of expression in the placenta during the first, second, and third trimester. Similarly, DPP4, which is translated into an intrinsic membrane glycoprotein, was highly expressed throughout gestation (Figure 1A).

Next, we analyzed the gene expression profile during gestation in placental tissues. We found 25 differentially expressed genes (DEGs) in the second trimester, and 687 DEGs in the third, when they were independently compared to the first trimester (Supplementary Table S1). All DEGs were divided into three clusters by the K-means clustering analysis (Supplementary Figure S1A). Cluster 1 includes genes that increase expression during pregnancy, and these genes enriched terms related to blood vessels morphogenesis, complement cascade, extracellular matrix organization, and cellular response to nitrogen compounds. Cluster 2 encompasses genes that increase expression specifically in the third trimesters, which are related to female pregnancy, growth hormone signaling pathway, homeostasis, and steroid biosynthetic process. Cluster 3 includes genes that decrease expression during pregnancy. These genes enriched terms related to cell division, sulfur compounds biosynthetic process, chromosomal segregation, PID MYC active pathway (Supplementary Figure S1B, Supplementary Table S2). The gene expression changes in the course of pregnancy revealed that the second and third trimesters enriched genes that are associated with the placental growth, mainly in the third trimester (Supplementary Figure S1C, Supplementary Table S3). Even with a high discrepancy between the number of DEGs in the second and third trimesters (25 and 687, respectively), we identified similarities in the enriched terms between gene clusters (Supplementary Figure S1D) and between the second and third trimesters of gestation (Supplementary Figure S1E).

We further asked whether the DEGs in the placenta during gestation are translated into proteins that potentially interact with SARS-CoV proteins. Considering that the current cases of SARS$\mathrm{CoV}-2$ placental infection are mainly related to the third trimester 
A

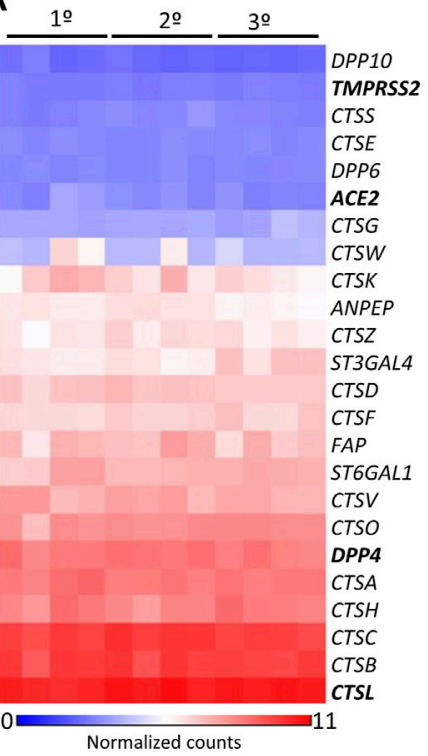

B

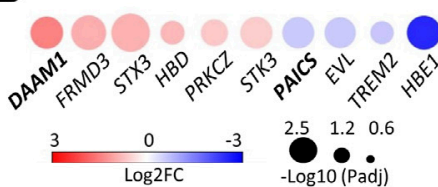

D Endo $(\mathrm{m})$ Endo $\mathrm{L}$

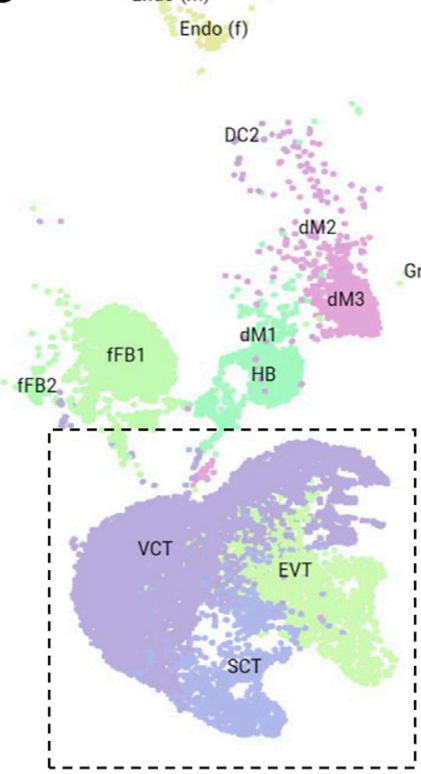

C

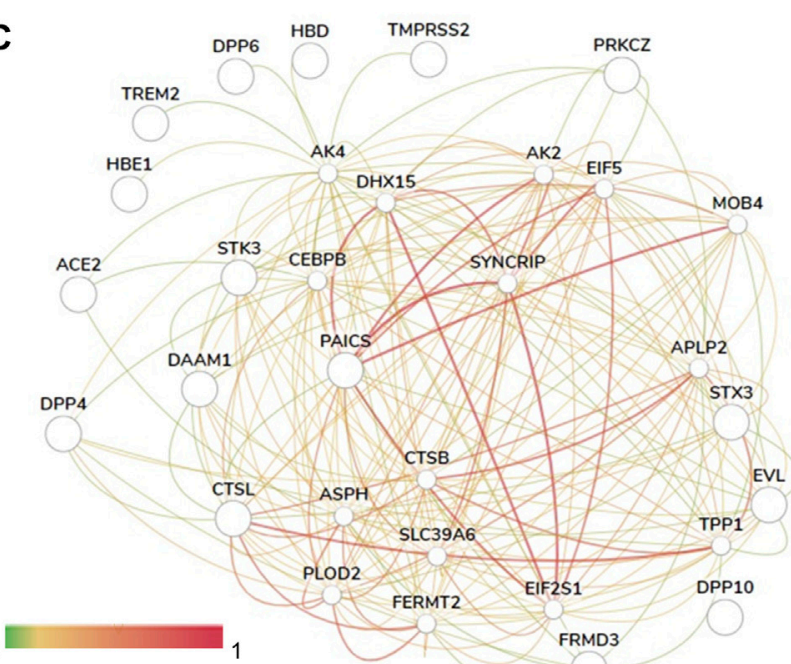

E

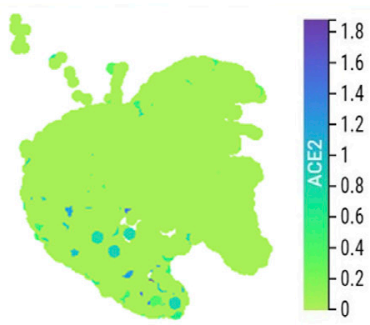

8
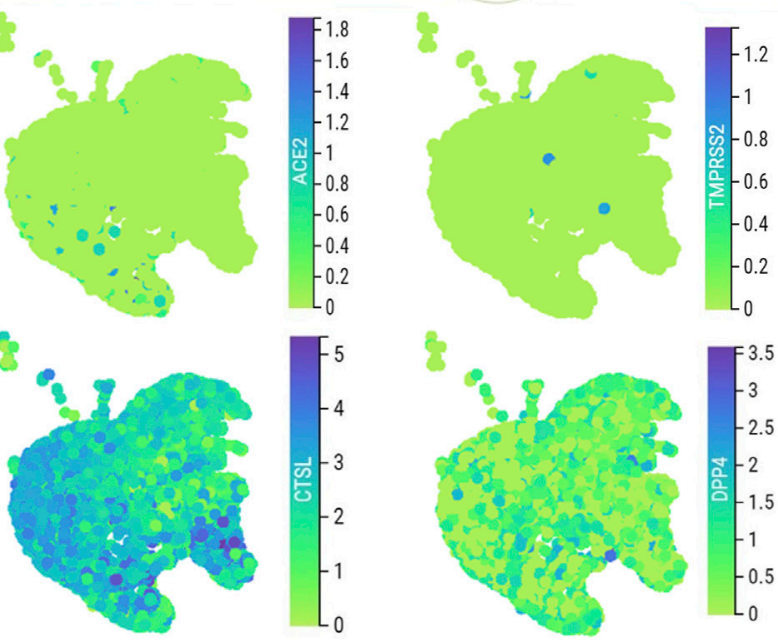

8

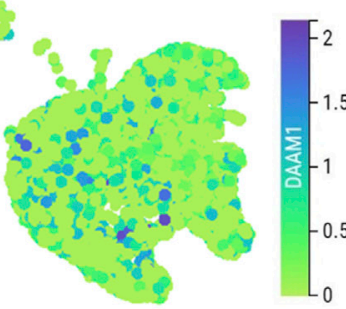

8

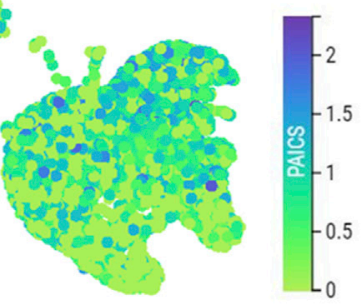

$\mathbf{F}$
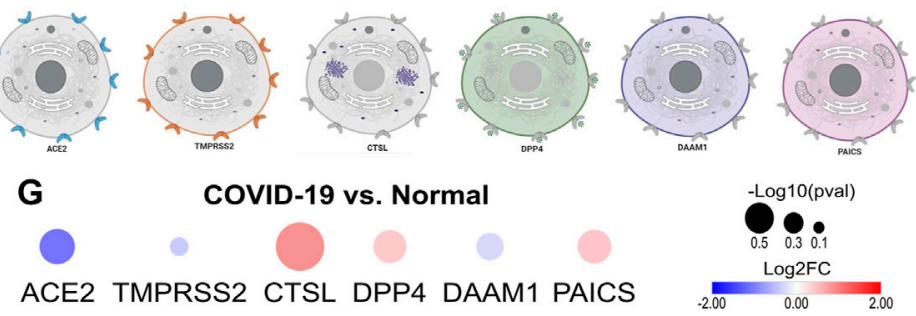

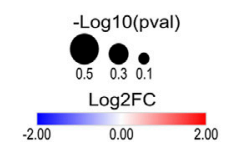

FIGURE 1 | The expression landscape of human placenta proteins potentially interacting with SARS-CoV-2. (A). Heatmap illustrating gene expression (counts RMA normalized) of SARS-CoV-2 interacting proteins from placental samples in the first, second and third trimester of gestation (GSE9984). (B). Heat-scatter plot presenting ten differentially expressed genes in the placenta that encode proteins potentially interacting with SARS-CoV as predicted by the P-HIPSter (http://phipster. org/) database. The color and size of the circles correspond to the log2FC and -log10 transformed FDR adjusted $p$-value, respectively. Fold change represents the gene expression of placenta samples from the third trimester of gestation compared with the first trimester. Bolded genes represent the six candidates selected for the further scRNA-seq evaluations. (C). Tissue-specific gene network of placenta proteins predicted to interact with coronaviruses. The network was generated using the (Continued) 
FIGURE 1 | humanbase (https://hb.flatironinstitute.org/) online tool. (D). Dimensionality reduction demonstrates different cell populations identified in five first-trimester human placental samples in a Uniform Manifold Approximation and Projection (UMAP) plot, by using scRNA-Seq data from Vento-Tormo et al., 2018. The images were generated using the COVID-19 Cell Atlas (https://www.covid19cellatlas.org) functionalities. (E). UMAP plots representing the gene expression (log-transformed normalized counts) of ACE2, TMPRSS2, CTSL, DPP4, DAAM1, and PAICS in human placental cells from the syncytiotrophoblast, villous cytotrophoblast, and extravillous trophoblast. The images were generated using COVID-19 Cell Atlas (https://www.covid19cellatlas.org) functionalities. (F). Cell illustrations depicting the protein localization of ACE2, TMPRSS2, CTSL, DPP4, DAAM1, and PAICS in cell compartments, as described in the Human Protein Atlas database (http://www. proteinatlas.org). Illustrations were created using BioRender App (https://biorender.com/). (G). Heat-scatter plot presenting gene expression of ACE2, TMPRSS2, CTSL, DPP4, DAAM1, and PAICS in the placenta from COVID-19 pregnant women compared to healthy pregnant (GSE17995). The color and size of the circles correspond to the log2FC and -log10 transformed $p$-values, respectively. Fold change represents the gene expression of placenta samples from the third trimester of gestation of COVID-19 women compared with matched third trimester uninfected women. RMA: Robust Multichip Average; FC: Fold Change; FDR: false discovery rate; Padj: adjusted p-value; SCT, syncytiotrophoblast; VCT, villous cytotrophoblast; EVT, extravillous trophoblast; HB, Hofbauer cells; FB: fibroblasts; dM, decidual macrophages; Endo, endothelial cells; I, Iymphatic; m, maternal; f, fetal.

of pregnancy (Patanè et al., 2020; Shanes et al., 2020), we selected the 687 DEGs in the placenta in the third trimester compared to the first trimester and, among them, 474 and 213 were up- and down-regulated, respectively (Supplementary Table S1). Next, we selected the human proteins potentially interacting with SARS-CoV using the P-HIPSter database. We found 32 virushost interacting proteins of SARS-CoV (Supplementary Table S4), and nine virus-host interacting proteins of the ZIKV (Supplementary Table S5). We also found that, from this list of virus-host interacting proteins, 10 DEGs (DAAM1, FRMD3, STX3, HBD, PRKCZ, CTK3, PAICS, EVL, TREM2, and HBE1) are translated into proteins that interact with SARS-CoV proteins, and one with the ZIKV (Supplementary Figure S1F,G). Among these 10 DEGs, six were up-regulated (DAAM1, FRMD3, STX3, $H B D, P R K C Z$, and CTK3) and four (PAICS, EVL, TREM2, and $H B E 1$ ) were down-regulated in the third trimester (Figure 1B). The gene DAAM1, which is translated into an intrinsic membrane glycoprotein implicated in cell motility, adhesion, cytokinesis, and cell polarity-showed the highest level of fold change in the third trimester of gestation compared to the first $(\log F C=1.43$; Figure 1B). The host-virus protein-protein interactions (PPI) predicted for these 10 DEGs presented a Likelihood Ratio (LR) > 100 (Supplementary Table S6), according to P-HIPSTer (Lasso et al., 2019). Noteworthy, PAICS transcript, which is translated into an enzyme that catalyzes the sixth and seventh steps of the novo purine biosynthesis, were predicted to interact with both SARS-CoV and ZIKV (Figure 1B, Supplementary Table S7). We observed a significant interaction of placental proteins predicted to interact with SARS-CoV-2 (based on the DEGs or not), and PAICS was also predicted to interact with other proteins in the PPI network with the highest degree (Figure 1C, Supplementary Table S8).

We next used single-cell RNA sequencing to analyze the expression of the 10 DEGs translated into proteins that potentially interact with SARS-CoV in human placental cells (Supplementary Figure $\mathbf{S 1 H}, \mathbf{I}$ ). We selected the genes ACE2, TMPRSS2, CTSL, DPP4, PAICS, and DAAM1 for further investigations using scRNA-seq transcriptome data from cells of the syncytiotrophoblast ( $n=1,144)$, villous cytotrophoblast ( $n=8,244)$, and extravillous trophoblast $(n=2,170)$ of nondisease human placental tissues, considering the potential relevance of these genes for SARS-CoV infection and replication in the organ (Figure 1D). We noticed that the expression of the classical SARS-CoV-2 entry receptor genes
(ACE2 and TMPRSS2) was minimally expressed in these cells. In contrast, potential non-canonical cell entry mediator genes DPP4 and CTSL, as well as the genes for the predicted virus-host interaction proteins DAAM1, and PAICS were expressed at higher levels (Figure 1E, Supplementary Figure S2A). We also looked for the co-expression of ACE2 and TMPRSS2 with DPP4, CTSL, DAAM1, and PAICS by using scRNA-Seq in these same cells (Figure 2). This analysis revealed that DPP4, CTSL, $D A A M 1$, and PAICS are co-expressed at high levels, while these genes are co-express at low levels with ACE2 and TMPRSS2 (Figure 2). Remarkably, we found only three cells co-expressing ACE2 and TMPRSS2. For this reason, we consider that DPP4, CTSL, DAAM1, and PAICS may represent candidates of an alternative route for SARS-CoV-2 infection in human placentas. We used The Human Protein Atlas to predict the subcellular location of proteins encoded by our candidates. DPP4 is predicted as intracellular, membrane, and secreted protein; CTSL is a protein located in the Golgi apparatus and additionally in vesicles; PAICS is a protein found in the cytosol while DAAM1 is in the plasma membrane and cytosol (Figure 1F). A recent published study investigated the gene expression alteration in the term placentas from COVID-19 pregnant women compared to uninfected (Lu-Culligan et al., 2021). We have accessed the DEGs identified by the authors, and we found that our candidate genes are not differentially expressed (Figure 1G). Moreover, DAAM1 and PAICS are expressed in the opposite direction to the one found during gestational ages (Figure 1B). Finally, we analyzed the gene expression profile of ACE2, TMPRSS2, CTSL, DPP4, $D A A M 1$, and PAICS genes on publicly available scRNA-Seq datasets of lung, liver, and thymus fetal tissues. CTSL, DPP4, and DAAM1 were found as highly expressed in fetal cells compared to ACE2 and TMPRSS2 in all tissues analyzed (Supplementary Figure S2).

\section{DISCUSSION}

Recent literature has shown placenta cells infected with SARSCoV-2 in pregnant women diagnosed with moderate to severe COVID-19 (Patanè et al., 2020; Penfield et al., 2020), with findings supporting the vertical transmission of SARS-CoV-2 in early and late pregnancy (Hosier et al., 2020; Patanè et al., 2020; Schwartz and Morotti, 2020; Shende et al., 2021; ValdespinoVázquez et al., 2021). However, few placenta cells express ACE2 

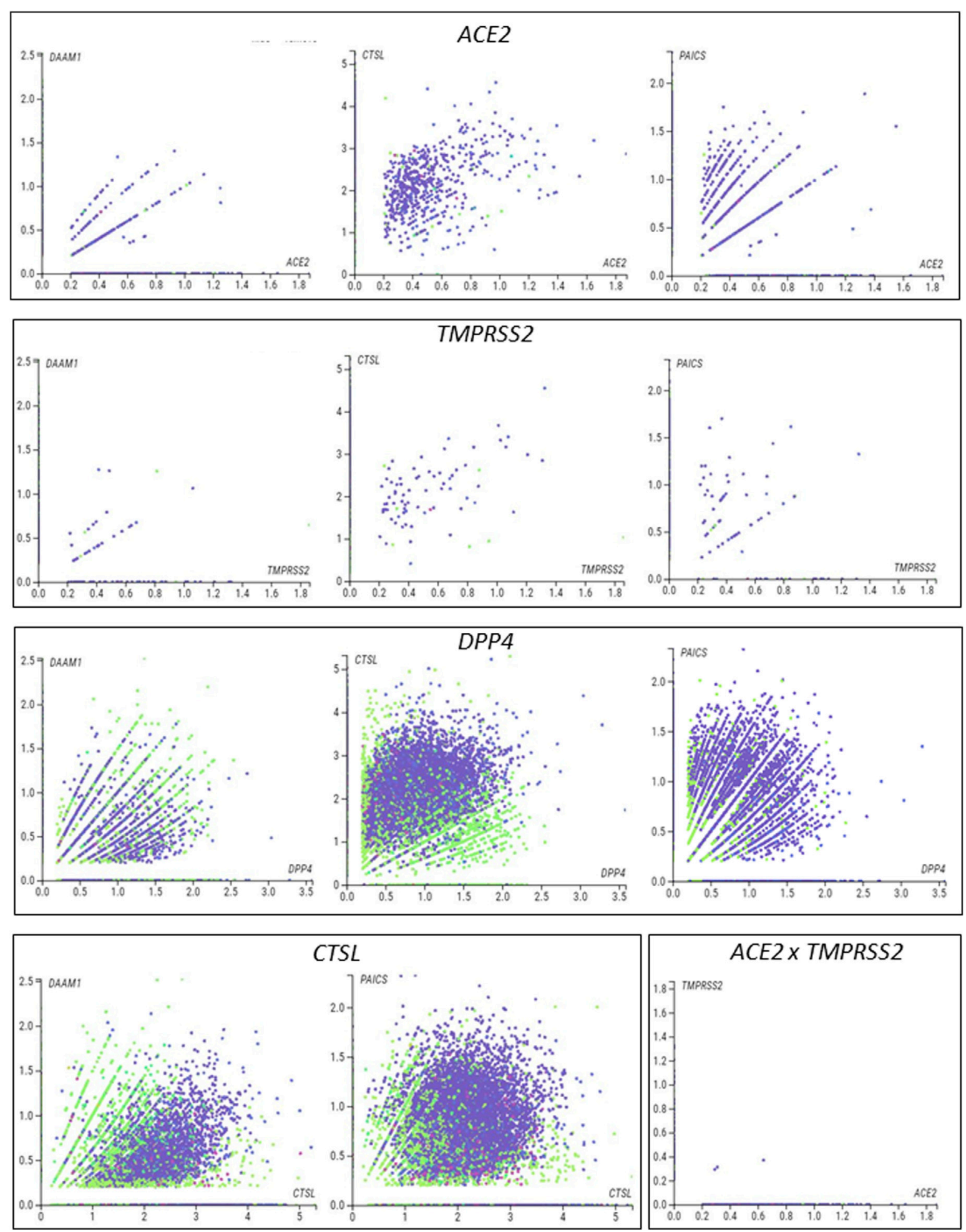

FIGURE 2 | Single-cell analysis of the human placenta indicates potential non-canonical routes of SARS-CoV-2. (A). Co-expression analysis of DAAM1, CTSL, DPP4, and PAICS with the canonical virus entry-associated genes ACE2 and TMPRSS2. (B). Co-expression analysis of DAAM1 and PAICS with the non-canonical virus entry genes DPP4 and CTLS. The images were generated using COVID-19 Cell Atlas (https://www.covid19cellatlas.org) functionalities. 
and TMPRSS2 (Pique-Regi et al., 2020; Sungnak et al., 2020) required for virus entry, raising the question of whether potential non-canonical molecular mechanisms may be involved in the biological cycle of the virus in these cells. Here, we demonstrated by transcriptomic analyses (microarray and scRNA-Seq) and in silico predictions of virus-host protein-protein interactions that, despite low-levels of ACE2 and TMPRSS2, villous trophoblast cells express high levels of the potential non-canonical cell-entry mediators DPP4 and CTSL. We also found changes in the expression of DAAM1 and PAICS genes that code for proteins predicted to interact with SARS-CoV proteins during pregnancy. These results provide evidence of potential host-virus PPI that may lead to SARS-CoV-2 infection and replication in the human placenta.

Firstly, we characterized the expression of SARS-CoV-2/ SARS-CoV entry-associated receptors and proteases genes, which revealed that DPP4 and CTSL are highly expressed in villous trophoblast cells during pregnancy. Similar to our findings, DPP4 and CTSL expression was already identified in first and second trimester human placenta single cells (Ashary et al., 2020). DPP4 has a high affinity to the SARS-CoV-2 spike receptor-binding domain, (Li et al., 2020), and critical DPP4 residues share the SARS-CoV-2-S/DPP4 binding as found for MERS-CoV-S/DPP4 (Hoffmann et al., 2020). Although it was demonstrated that DPP4 is not a SARS-CoV-2 entry receptors in BHK-21 cells (Hoffmann et al., 2020), more studies are necessary to address the role of DPP4 in placental cells. In a pioneer study based on SARS-CoV cell entry mechanisms, (Simmons et al., 2005) described a three-step method for host-virus membrane fusion, involving receptor-binding and induced conformational changes in S glycoprotein with subsequent CTSL proteolysis and activation of membrane fusion within endosomes. During the COVID-19 pandemic, it has been further demonstrated that SARS-CoV-2 can use CTSB and CTSL as well as TMPRSS2 for priming host cells (Hoffmann et al., 2020; Ou et al., 2020). These data provide evidence that CTSL is a potentially promising treatment for COVID-19 by blocking coronavirus host cell entry and intracellular replication (Liu et al., 2020). Secondly, we analyzed whether placental development and growth are associated with transcriptional changes in proteins that potentially interact with SARS-CoV. Among the transcripts translated into proteins predicted as interacting with SARSCoV, DAAM1 increased with placental development and growth. DAAM1 was previously identified as a regulator of bacteria phagocytosis by regulating filopodia formation and phagocytic uptake in primary human macrophages (Hoffmann et al., 2014). We predicted that DAAM1 potentially interacts with the viral protein encoded from open reading frame 8 (ORF8) and the hypothetical protein sars7a of SARS-CoV. The SARS-CoV ORF8 shares the lowest homology with all SARS-CoV-2 proteins; however, it was previously demonstrated that SARS-CoV-2 ORF8 expression was able to selectively target MHC-I for lysosomal degradation by an autophagy-dependent mechanism in different cell types (Zhang et al., 2020). We found that the PAICS transcript, which is also translated into a protein predicted as interacting with the Nsp3 of SARS-CoV, decreased its levels with placental development and growth. PAICS is an enzyme of the de novo purine biosynthesis pathway, which was previously predicted as having a putative interaction with the human influenza A virus (IAV) nucleoprotein and was up-regulated during IAV infection (Generous et al., 2014). The putative interaction of Nsp3 with PAICS is relevant. Nsp3 is one of the 16 non-structural proteins in the replicase ORF1ab gene of SARSCoV and SARS-CoV-2 (Enjuanes, 2005; Wu et al., 2020), and binds to virus RNA and proteins, including the nucleocapsid protein (Lei et al., 2018). Considering the low levels of ACE2 and TMPRSS2 that we found in villous trophoblast cells, our results provide evidence of alternative cell-entry mediators in the placenta. Moreover, the description of the potential interaction between host and SARS-CoV-2 may provide insights into the mechanisms of placental infection in women with COVID-19.

Cellular and molecular composition play a role in placenta infection and intrauterine transmission of viruses (Koi et al., 2001; Barbeito-Andrés et al., 2020). Thus, we finally used singlecell analysis of the syncytiotrophoblast, villous cytotrophoblast, and extravillous trophoblasts-the fetal component of the placenta-that confirmed the low levels of expression of the canonical entry receptor ACE2 and TMPRSS2 genes (PiqueRegi et al., 2020; Sungnak et al., 2020). Conversely, we found that these cells express high-levels of DPP4 and CTSL, two potential mediators of SARS-Cov-2 host cell entry (Hoffmann et al., 2020; Li et al., 2020) that may contribute to the SARS-CoV2 infection (Hosier et al., 2020; Patanè et al., 2020; Penfield et al., 2020). It is essential to highlight that our scRNA-Seq analysis showed that the non-canonical DPP4 and CTSL entry genes (Hoffmann et al., 2020; Li et al., 2020) are highly co-expressed in syncytiotrophoblast, villous cytotrophoblast, and extravillous trophoblasts cells. DPP4 and CTSL expression landscape in different tissues were extensively investigated in scRNA-seq study (Singh et al., 2020). However, there was no evidence of $D A A M 1$ and PAICS role. We found that the transcripts DAAM1 and PAICS, which are translated into proteins predicted as potentially interacting with SARS-CoV-2, were also highly coexpressed with DPP4 and CTSL. These results demonstrate that, although ACE2 and TMPRSS2 are poorly expressed in the placenta, other mediators that potentially interact with the virus are highly co-expressed in villous trophoblast cells and, therefore, may represent a valuable alternative route for infection and viral replication.

Although our in-silico analyses are a starting point, they have limitations. We reanalyzed published placenta datasets with a limited number of samples (microarray $=12$ individuals, and scRNA-seq $=5$ individuals) and, consequently, validations in a large cohort of samples must be performed. We understand that under sampling in transcriptomic data are a limitation and statistical analysis as imbalanced learn and ablation tests would benefit our methodology, however, our main results are supported by the relevant literature. Moreover, the predicted interactions presented here should also be experimentally validated in infected cells or placenta to circumvent these limitations. Moreover, the analyses of single-cell data should be conducted for the entire period of gestation, considering that we only evaluated single-cell data from the first trimester of pregnancy. The detection of SARS-CoV-2 in the placenta needs 
to be performed in a large cohort of pregnant women with COVID-19, including asymptomatics. Considering the vertical transmission of SARS-CoV-2, the follow-up of newborns from mothers with COVID-19 during pregnancy should be necessary since, if it occurs even in the non-asymptomatic, the long-term consequences are mostly unknown.

In conclusion, despite low levels of ACE2 and TMPRSS2, our analyses demonstrate that villous trophoblast cells express high levels of potential non-canonical cell-entry mediators DDP4 and CTSL. We also found changes in the expression of the DAAM1 and PAICS genes coding for proteins predicted to interact with SARS-CoV proteins during pregnancy. These results provide new insight into the interaction between SARS-CoV-2 and the host proteins and indicate that coronaviruses may use multiple mediators for virus infection and replication.

\section{MATERIALS AND METHODS}

\section{Differential Expression Analysis of the Placenta During Pregnancy Trimesters}

We reanalyzed microarray data from villous trophoblast tissues of first (45-59 days, $n=4)$, second trimester (109-115 days, $n=4)$, and third trimesters $(n=4)$ of uncomplicated pregnancies, available in Gene Expression Omnibus (GEO), under the accession number GSE9984 (Mikheev et al., 2008). Next, we identified the DEGs during the pregnancy, by comparing the second trimester and third trimester with the first trimester using the GEO2R tool (https://www.ncbi.nlm.nih.gov/geo/geo2r). We applied the default settings of GEO2R to perform transcriptome analysis on original submitter-supplied processed data tables (RMA normalized signal $\log 2$ ) using the GEOquery and limma $\mathrm{R}$ packages from the Bioconductor project. Genes with $\log 2$ of Fold Change $\geq|0.5|$ and False Discovery Rate $(\mathrm{FDR})<0.05$ were considered as DEGs. We further grouped the gene expression profiles during pregnancy by using the K-means clustering analysis based on One Minus Pearson Correlation (Robust Multi-array Average, RMA, normalization $\log 2$, K-means $=3$ ).

\section{Enrichment Analysis}

We used the Metascape tool (https://metascape.org) (Zhou et al., 2019) to perform functional enrichment analysis of the genes lists generated in the clustering and differential expression analyses.

\section{Placenta Proteins That Potentially Interact With Coronaviruses and Zika Virus}

SARS-CoV-2 cell entry mediators were selected for first screening using the human proteins already described in the COVID-19 Cell Atlas (https://www.covid19cellatlas.org/) and the literature (Hoffmann et al., 2020; Li et al., 2020; Sungnak et al., 2020; Zhou et al., 2020). Next, we used gene expression data to generated a list of PPIs that potentially interact with human coronaviruses and the ZIKV from the Pathogen-Host
Interactome Prediction using Structure Similarity (P-HIPSTer, http://phipster.org/) database (Hosier et al., 2020) (Supplementary Tables S4,S5, respectively). SARS-CoV was included in the analysis considering the evolutionary relationship between the novel SARS-CoV-2 (Zhou et al., 2020), and ZIKV considering its impact on placental infection and fetal microcephaly (Pierson and Diamond, 2018; Barbeito-Andrés et al., 2020).

\section{Tissue-specific Gene Networks of Potential Virus-Host Placenta Interactome}

The humanbase webtool (https://hb.flatironinstitute.org) (Greene et al., 2015) was used to generate the tissue-specific gene network of placental genes coding for proteins that potentially interact with SARS-CoV-2 based on P-HIPSTER or that we identified as either associated with COVID-19 or that we hypothesized may be associated with the disease, based on the literature (Supplementary Table S8). We considered coexpression, interaction, transcriptional factor binding, Gene Set Enrichment Analysis (GSEA) perturbations as active interaction sources, applying minimum interaction confidence of 0.07 , and a maximum number of 15 genes.

\section{Single-Cell Transcriptome Analysis of Human Placentas and Fetal Tissues}

We used the functionalities of the COVID-19 Cell Atlas (https://www.covid19cellatlas.org) to explore single-cell RNA sequencing (scRNA-Seq) data from five samples from first-trimester placentas, previously described by VentoTormo et al. (2018). The processed scRNA-seq data (logtransformed normalized counts) of placental genes potentially interacting with SARS-CoVs was visualized in placental cell types using the "interactive viewer" developed by cellxgene v0.15.0 (https://cellxgene.cziscience.com) by the Chan Zuckerberg Initiative available at https://cellxgene. cziscience.com/d/fetal_maternal_interface_10x-22.cxg/. We also characterized the gene expression profile of canonical and non-canonical placental mediators of viruses interaction per cell using cellxgene v0.15.0 bar plots. The placental singlecell transcriptome is available for re-analysis at Human Cell Atlas Data Portal (https://data.humancellatlas.org) or E-MTAB-6701 (EMBL-EBI, ArrayExpress; https://www.ebi. ac.uk/arrayexpress). We applied this same strategy to investigate the gene expression of our candidates using scRNA-seq in human fetal tissues: lung, liver, and thymus (ArrayExpress; E-MTAB-8221, E-MTAB-7407, and E-MTAB8581, respectively. Last accessed June 2020.

\section{Placenta RNA Sequencing From Pregnant Women With COVID-19}

We downloaded the list of differentially expressed genes from 3rd trimester placental villi of pregnant women with COVID-19 $(n=$ 5) compared with uninfected control individuals matched for maternal age, gestational age, maternal comorbidities, and mode 
of delivery $(n=3$ ), publicly available at GSE17995 (Lu-Culligan et al., 2021). The authors conducted the differential expression analysis using DESeq2 v1.24.0 with default parameters, and we downloaded the CSV file from GEO.

\section{Data Representation and Analysis}

Morpheus (https://software.broadinstitute.org/morpheus/) was used to generate the heatmaps and to perform the clustering analyses. The Venn diagram was constructed using the Van de Peer Lab software (http://bioinformatics.psb.ugent.be/webtools/Venn/). Biorender was used to design cell images (https://app.biorender.com/).

\section{DATA AVAILABILITY STATEMENT}

The datasets presented in this study can be found in online repositories. The names of the repository/repositories and accession numbers can be found in the article/Supplementary Material.

\section{ETHICS STATEMENT}

This study was a retrospective analysis based on the reanalysis of existing publicly available datasets available from the GEO (https://www.ncbi.nlm.nih.gov/geo/; GSE9984 and GSE17995), Human Cell Atlas Data Portal (https://data.humancellatlas. org/; Vento-Tormo et al. (2018)), and EMBL-EBI, Express (https://www.ebi.ac.uk/arrayexpress; E-MTAB-6701, E-MTAB8221, E-MTAB-7407, and E-MTAB-8581). Therefore, ethics approval and patient consent are not required.

\section{REFERENCES}

Ashary, N., Bhide, A., Chakraborty, P., Colaco, S., Mishra, A., Chhabria, K., et al. (2020). Single-Cell RNA-Seq Identifies Cell Subsets in Human Placenta that Highly Expresses Factors Driving Pathogenesis of SARS-CoV-2. Front. Cel Dev. Biol. 8, 783. doi:10.3389/fcell.2020.00783

Barbeito-Andrés, J., Pezzuto, P., Higa, L. M., Dias, A. A., Vasconcelos, J. M., Santos, T. M. P., et al. (2020). Congenital Zika Syndrome Is Associated with Maternal Protein Malnutrition. Sci. Adv. 6, eaaw6284. doi:10.1126/sciadv.aaw6284

Celik, O., Saglam, A., Baysal, B., Derwig, I. E., Celik, N., Ak, M., et al. (2020). Factors Preventing Materno-Fetal Transmission of SARS-CoV-2. Placenta 97, 1-5. doi:10.1016/j.placenta.2020.05.012

Chen, H., Guo, J., Wang, C., Luo, F., Yu, X., Zhang, W., et al. (2020). Clinical Characteristics and Intrauterine Vertical Transmission Potential of COVID-19 Infection in Nine Pregnant Women: A Retrospective Review of Medical Records. The Lancet 395, 809-815. doi:10.1016/S0140-6736(20) 30360-3

Constantino, F. B., Cury, S. S., Nogueira, C. R., Carvalho, R. F., and Justulin, L. A. (2020). Prediction of Non-Canonical Routes for SARS-CoV-2 Infection in Human Placenta Cells. bioRxiv. doi:10.1101/2020.06.12.148411

Dong, E., Du, H., and Gardner, L. (2020a). An Interactive Web-Based Dashboard to Track COVID-19 in Real Time. Lancet Infect. Dis. 20, 533-534. doi:10.1016/ S1473-3099(20)30120-1

Dong, L., Tian, J., He, S., Zhu, C., Wang, J., Liu, C., et al. (2020b). Possible Vertical Transmission of SARS-CoV-2 from an Infected Mother to Her Newborn. JAMA 323 (18), 1846-1848. doi:10.1001/jama.2020.4621

Generous, A., Thorson, M., Barcus, J., Jacher, J., Busch, M., and Sleister, H. (2014). Identification of Putative Interactions between Swine and Human Influenza A

\section{AUTHOR CONTRIBUTIONS}

Conceptualization, FC, RC, LJ; methodology, FC, RC, SC, LJ; formal analysis, $\mathrm{FC}, \mathrm{CN}, \mathrm{RC}, \mathrm{SC}, \mathrm{LJ}$; investigation, $\mathrm{FC}, \mathrm{CN}, \mathrm{RC}$, SC, LJ; resources, RC, LJ; data curation, FC, CN, RC, SC, LJ; writing-original draft preparation, FC, RC, SC, LJ.; writingreview and editing, all authors; supervision, RC, LJ; project administration, RC, LJ; funding acquisition, RC, LJ.

\section{FUNDING}

This research was supported by the National Council for Scientific and Technological Development, CNPq (Process 311530/2019-2 to RC, and Process 310663/2018-0 to LJ, and scholarship \#870415/ 1997-2 to SC), and by Fundação de Amparo à Pesquisa do Estado de São Paulo (Process 2012/13961-6 to RC, Process 2017/01063-7 to LJ, and scholarship 2017/08716-6 to FBC).

\section{ACKNOWLEDGMENTS}

This manuscript has been released as a pre-print at bioRxiv (Constantino et al., 2020).

\section{SUPPLEMENTARY MATERIAL}

The Supplementary Material for this article can be found online at: https://www.frontiersin.org/articles/10.3389/fmolb.2021.614728/ full\#supplementary-material

Virus Nucleoprotein and Human Host Proteins. Virol. J. 11, 228. doi:10.1186/ s12985-014-0228-6

Greene, C. S., Krishnan, A., Wong, A. K., Ricciotti, E., Zelaya, R. A., Himmelstein, D. S., et al. (2015). Understanding Multicellular Function and Disease with Human Tissue-Specific Networks. Nat. Genet. 47, 569-576. doi:10.1038/ ng.3259

Hashimoto, Y., Sheng, X., Murray-Nerger, L. A., and Cristea, I. M. (2020). Temporal Dynamics of Protein Complex Formation and Dissociation during Human Cytomegalovirus Infection. Nat. Commun. 11, 806. doi:10.1038/s41467-020-14586-5

Hoffmann, A. K., Naj, X., and Linder, S. (2014). Daam1 Is a Regulator of Filopodia Formation and Phagocytic Uptake of Borrelia Burgdorferi by Primary Human Macrophages. FASEB j. 28, 3075-3089. doi:10.1096/fj.13-247049

Hoffmann, M., Kleine-Weber, H., Schroeder, S., Krüger, N., Herrler, T., Erichsen, S., et al. (2020). SARS-CoV-2 Cell Entry Depends on ACE2 and TMPRSS2 and Is Blocked by a Clinically Proven Protease Inhibitor. Cell 181, 271-280. e8. doi:10.1016/j.cell.2020.02.052

Hosier, H., Farhadian, S., Morotti, R. A., Deshmukh, U., Lu-Culligan, A., Campbell, K. H., et al. (2020). SARS-CoV-2 Infection of the Placenta. medRxiv. doi:10.1101/2020.04.30.20083907

Kimberlin, D. W., and Stagno, S. (2020). Can SARS-CoV-2 Infection Be Acquired In Utero?: More Definitive Evidence Is Needed. JAMA 323 (18), 1788-1789. doi:10.1001/jama.2020.4868

Koi, H., Zhang, J., and Parry, S. (2001). The Mechanisms of Placental Viral Infection. Ann. N.Y Acad. Sci. 943, 148-156. doi:10.1111/j.17496632.2001.tb03798.x

Lasso, G., Mayer, S. V., Winkelmann, E. R., Chu, T., Elliot, O., Patino-Galindo, J. A., et al. (2019). A Structure-Informed Atlas of Human-Virus Interactions. Cell 178, 1526-1541. e16. doi:10.1016/j.cell.2019.08.005 
Lei, J., Kusov, Y., and Hilgenfeld, R. (2018). Nsp3 of Coronaviruses: Structures and Functions of a Large Multi-Domain Protein. Antiviral Res. 149, 58-74. doi:10.1016/j.antiviral.2017.11.001

L. Enjuanes (Editor) (2005). Coronavirus Replication and Reverse Genetics (Berlin, Heidelberg: Springer Berlin Heidelberg). doi:10.1007/b138038

Letko, M., Marzi, A., and Munster, V. (2020). Functional Assessment of Cell Entry and Receptor Usage for SARS-CoV-2 and Other Lineage B Betacoronaviruses. Nat. Microbiol. 5, 562-569. doi:10.1038/s41564-020-0688-y

Li, Y., Zhang, Z., Yang, L., Lian, X., Xie, Y., Li, S., et al. (2020). The MERS-CoV Receptor DPP4 as a Candidate Binding Target of the SARS-CoV-2 Spike. iScience 23, 101160. doi:10.1016/j.isci.2020.101160

Liu, T., Luo, S., Libby, P., and Shi, G.-P. (2020). Cathepsin L-Selective Inhibitors: A Potentially Promising Treatment for COVID-19 Patients. Pharmacol. Ther. 213, 107587. doi:10.1016/j.pharmthera.2020.107587

Lu-Culligan, A., Chavan, A. R., Vijayakumar, P., Irshaid, L., Courchaine, E. M., Milano, K. M., et al. (2021). Maternal Respiratory SARS-CoV-2 Infection in Pregnancy Is Associated with a Robust Inflammatory Response at the MaternalFetal Interface. Med 2, 591-610. e10. doi:10.1016/j.medj.2021.04.016

Mikheev, A. M., Nabekura, T., Kaddoumi, A., Bammler, T. K., Govindarajan, R., Hebert, M. F., et al. (2008). Profiling Gene Expression in Human Placentae of Different Gestational Ages: An OPRU* Network and UW SCOR Study. Reprod. Sci. 15, 866-877. doi:10.1177/1933719108322425

Ou, X., Liu, Y., Lei, X., Li, P., Mi, D., Ren, L., et al. (2020). Characterization of Spike Glycoprotein of SARS-CoV-2 on Virus Entry and its Immune Cross-Reactivity with SARS-CoV. Nat. Commun. 11, 1620. doi:10.1038/s41467-020-15562-9

Patanè, L., Morotti, D., Giunta, M. R., Sigismondi, C., Piccoli, M. G., Frigerio, L., et al. (2020). Vertical Transmission of Coronavirus Disease 2019: Severe Acute Respiratory Syndrome Coronavirus 2 RNA on the Fetal Side of the Placenta in Pregnancies with Coronavirus Disease 2019-positive Mothers and Neonates at Birth. Am. J. Obstet. Gynecol. MFM 2, 100145. doi:10.1016/j.ajogmf.2020.100145

Penfield, C. A., Brubaker, S. G., Limaye, M. A., Lighter, J., Ratner, A. J., Thomas, K. M., et al. (2020). Detection of Severe Acute Respiratory Syndrome Coronavirus 2 in Placental and Fetal Membrane Samples. Am. J. Obstet. Gynecol. MFM 2, 100133. doi:10.1016/j.ajogmf.2020.100133

Pereira, L. (2018). Congenital Viral Infection: Traversing the Uterine-Placental Interface. Annu. Rev. Virol. 5, 273-299. doi:10.1146/annurev-virology-092917-043236

Pierson, T. C., and Diamond, M. S. (2018). The Emergence of Zika Virus and its New Clinical Syndromes. Nature 560, 573-581. doi:10.1038/s41586-0180446-y

Pique-Regi, R., Romero, R., Tarca, A. L., Luca, F., Xu, Y., Alazizi, A., et al. (2020). Does the Human Placenta Express the Canonical Cell Entry Mediators for SARS-CoV-2? eLife 9, e58716. doi:10.7554/eLife.58716

Qadri, F., and Mariona, F. (2020). Pregnancy Affected by SARS-CoV-2 Infection: A Flash Report from Michigan. J. Maternal-Fetal Neonatal Med. ahead of print. doi:10.1080/14767058.2020.1765334

Schoenmakers, S., Snijder, P., Verdijk, R. M., Kuiken, T., Kamphuis, S. S. M., Koopman, L. P., et al. (2020). SARS-CoV-2 Placental Infection and Inflammation Leading to Fetal Distress and Neonatal Multi-Organ Failure in an Asymptomatic Woman. medRxiv. doi:10.1101/2020.06.08.20110437

Schwartz, D. A., and Graham, A. L. (2020). Potential Maternal and Infant Outcombes from Coronavirus 2019-nCoV (SARS-CoV-2) Infecting Pregnant Women: Lessons from SARS, MERS, and Other Human Coronavirus Infections. Viruses 12, 194. doi:10.3390/v12020194

Schwartz, D. A., and Morotti, D. (2020). Placental Pathology of COVID-19 with and without Fetal and Neonatal Infection: Trophoblast Necrosis and Chronic Histiocytic Intervillositis as Risk Factors for Transplacental Transmission of SARS-CoV-2. Viruses 12, 1308. doi:10.3390/v12111308

Shanes, E. D., Mithal, L. B., Otero, S., Azad, H. A., Miller, E. S., and Goldstein, J. A. (2020). Placental Pathology in COVID-19. Am. J. Clin. Pathol. 154, 23-32. doi:10.1093/ajcp/aqaa089

Shende, P., Gaikwad, P., Gandhewar, M., Ukey, P., Bhide, A., Patel, V., et al. (2021). Persistence of SARS-CoV-2 in the First Trimester Placenta Leading to
Transplacental Transmission and Fetal Demise from an Asymptomatic Mother. Hum. Reprod. 36, 899-906. doi:10.1093/humrep/deaa367

Simmons, G., Gosalia, D. N., Rennekamp, A. J., Reeves, J. D., Diamond, S. L., and Bates, P. (2005). Inhibitors of Cathepsin L Prevent Severe Acute Respiratory Syndrome Coronavirus Entry. Proc. Natl. Acad. Sci. 102, 11876-11881. doi:10.1073/pnas.0505577102

Singh, M., Bansal, V., and Feschotte, C. (2020). A Single-Cell RNA Expression Map of Human Coronavirus Entry Factors. Cel Rep. 32, 108175. doi:10.1016/ j.celrep.2020.108175

Sungnak, W., Huang, N., Huang, N., Bécavin, C., Berg, M., Queen, R., et al. (2020). SARS-CoV-2 Entry Factors Are Highly Expressed in Nasal Epithelial Cells Together with Innate Immune Genes. Nat. Med. 26, 681-687. doi:10.1038/ s41591-020-0868-6

Valdespino-Vázquez, M. Y., Helguera-Repetto, C. A., León-Juárez, M., Villavicencio-Carrisoza, O., Flores-Pliego, A., Moreno-Verduzco, E. R., et al. (2021). Fetal and Placental Infection with SARS-CoV-2 in Early Pregnancy. J. Med. Virol. 93, 4480-4487. doi:10.1002/jmv.26965

Vento-Tormo, R., Efremova, M., Botting, R. A., Turco, M. Y., Vento-Tormo, M., Meyer, K. B., et al. (2018). Single-Cell Reconstruction of the Early MaternalFetal Interface in Humans. Nature 563, 347-353. doi:10.1038/s41586-0180698-6

Williamson, E. J., Walker, A. J., Bhaskaran, K., Bacon, S., Bates, C., Morton, C. E., et al. (2020). Factors Associated with COVID-19-related Death Using OpenSAFELY. Nature 584, 430-436. doi:10.1038/s41586-020-2521-4

Wu, F., Zhao, S., Yu, B., Chen, Y.-M., Wang, W., Song, Z.-G., et al. (2020). A New Coronavirus Associated with Human Respiratory Disease in China. Nature 579, 265-269. doi:10.1038/s41586-020-2008-3

Yang, Z., and Liu, Y. (2020). Vertical Transmission of Severe Acute Respiratory Syndrome Coronavirus 2: A Systematic Review. Am. J. Perinatol 37, 1055-1060. doi:10.1055/s-0040-1712161

Zeng, H., Xu, C., Fan, J., Tang, Y., Deng, Q., Zhang, W., et al. (2020). Antibodies in Infants Born to Mothers with COVID-19 Pneumonia. JAMA 323 (18), 1848-1849. doi:10.1001/jama.2020.4861

Zhang, Y., Zhang, J., Chen, Y., Luo, B., Yuan, Y., Huang, F., et al. (2020). The ORF8 Protein of SARS-CoV-2 Mediates Immune Evasion through Potently Downregulating MHC-I. bioRxiv. doi:10.1101/ 2020.05.24.111823

Zhou, P., Yang, X.-L., Wang, X.-G., Hu, B., Zhang, L., Zhang, W., et al. (2020). A Pneumonia Outbreak Associated with a New Coronavirus of Probable Bat Origin. Nature 579, 270-273. doi:10.1038/s41586-020-2012-7

Zhou, Y., Zhou, B., Pache, L., Chang, M., Khodabakhshi, A. H., Tanaseichuk, O., et al. (2019). Metascape Provides a Biologist-Oriented Resource for the Analysis of Systems-Level Datasets. Nat. Commun. 10, 1523. doi:10.1038/s41467-01909234-6

Conflict of Interest: The authors declare that the research was conducted in the absence of any commercial or financial relationships that could be construed as a potential conflict of interest.

Publisher's Note: All claims expressed in this article are solely those of the authors and do not necessarily represent those of their affiliated organizations, or those of the publisher, the editors and the reviewers. Any product that may be evaluated in this article, or claim that may be made by its manufacturer, is not guaranteed or endorsed by the publisher.

Copyright (c) 2021 Constantino, Cury, Nogueira, Carvalho and Justulin. This is an open-access article distributed under the terms of the Creative Commons Attribution License (CC BY). The use, distribution or reproduction in other forums is permitted, provided the original author(s) and the copyright owner(s) are credited and that the original publication in this journal is cited, in accordance with accepted academic practice. No use, distribution or reproduction is permitted which does not comply with these terms. 